\title{
HIGH-RESOLUTION ESTIMATION OF IONOSPHERIC PHASE SCREENS THROUGH SEMI-FOCUSING PROCESSING
}

\author{
Giorgio Gomba, Michael Eineder, Alessandro Parizzi and Richard Bamler
}

\author{
Remote Sensing Technology Institute, German Aerospace Center (DLR), Wessling, Germany
}

\begin{abstract}
Ionosphere irregularities along the synthetic aperture generate shifts and blurring that cause decorrelation. In this paper it is shown how, by partially focusing SAR images to the height of the ionosphere, it is possible to reduce the ionospheric azimuth effects and increase the coherence. This permits, even in case of turbulent ionosphere, to obtain better accuracies when separating the deformations phase from the ionospheric phase using the delta-k split-band interferometry method.
\end{abstract}

Index Terms - InSAR, SAR ionospheric effects, ionosphere estimation, ionosphere scintillation

\section{INTRODUCTION}

L-band remote sensing systems, like the future Tandem-L mission ([1]), are disrupted by the ionized upper part of the atmosphere called ionosphere. Ionospheric effects have to be estimated and corrected in order to fulfill the scientific requirements of the mission ([2]). This work concentrates on the effects that a ionosphere, which is unstable in the flight path direction, has on SAR images and interferograms. We then demonstrate a technique to eliminate these effects and produce high-resolution ionosphere estimates.

The ionosphere is a region of the upper atmosphere composed by gases that are ionized by the solar radiation. For this study we approximate the ionosphere by a thin layer at the altitude $H_{i}$ of the peak of its vertical profile. The effects of the ionosphere on a SAR image are:

- phase advance and range delay,

- range defocus,

- azimuth shift,

- azimuth defocus.

Azimuth defocus happens in particular when the correlation length of the ionospheric variations along the flight path of the satellite is shorter than the synthetic aperture length. Ionospheric variations change the impulse response function lowering the coherence and thus the interferogram accuracy.

A method to reduce the effects of an unstable ionosphere, called multi-squint interferometry, is presented in [3], however, the resolution inside the ionosphere is limited by the system parameters and geometry. In [4], it is suggested to defocus SAR images to the ionospheric height prior to apply
Faraday rotation estimation for measuring the ionosphere, to reduce the effects that ionosphere has on the impulse response function. In this work we demonstrate how, by partially focusing the images, it is actually possible to cancel or reduce the azimuth effects. The coherence is then increased and the estimation of the ionospheric and topographic phase becomes more accurate.

\section{IONOSPHERE IN INTERFEROGRAMS}

We neglect the range dimension to simplify the analysis. The geometry is represented in Figure 2 where $\psi(x)$ is the iono-

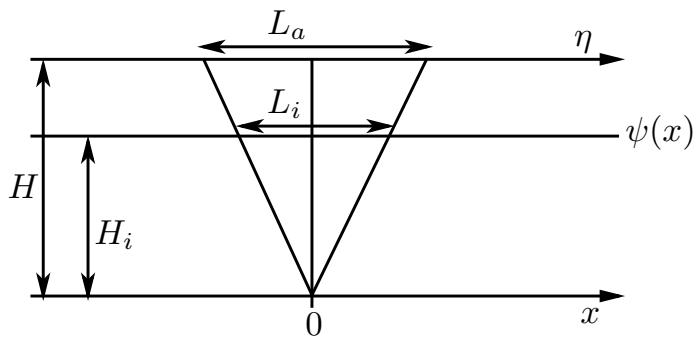

Fig. 1. System geometry, $x$ is the ground coordinate and $\eta$ is the satellite position. The ionosphere is modeled as a single layer so that $\psi(x)$ is the ionospheric phase screen.

spheric phase screen, $H$ is the height of the satellite orbit, $H_{i}$ is the single layer model height, $x$ is the ground coordinate and $\eta$ the orbit coordinate.

When a single scatter is located in $x=0$ an azimuth line of raw data collected by the satellite at the orbit position $\eta$ can be written as

$$
s_{r}(\eta)=a_{0} e^{j \alpha_{0}} \operatorname{rect}\left(\frac{\eta}{L_{a}}\right) e^{j \pi K \eta^{2}} e^{j \psi\left(\eta \frac{H_{i}}{H}\right)}
$$

where $a_{0}$ is the scatter reflectivity, $L_{a}$ is the aperture length, $K=2 / \lambda R_{0}$ the azimuth chirp rate and $\psi(x)$ the ionospheric phase screen. The standard azimuth focusing is a convolution 
with the conjugated chirp:

$$
\begin{aligned}
s_{f}(\eta) & =\int s_{r}(u) \operatorname{rect}\left(\frac{\eta-u}{L_{a}}\right) e^{-j \pi K(\eta-u)^{2}} d u \\
= & \int \operatorname{rect}\left(\frac{u}{L_{a}}\right) \operatorname{rect}\left(\frac{t-u}{L_{a}}\right) e^{j \psi\left(u \frac{H_{i}}{H}\right)} e^{-j 2 \pi K \eta u} d u .
\end{aligned}
$$

We notice that if the ionosphere remains stable for $-L_{i} / 2<$ $\eta<L_{i} / 2$, where $L_{i}$ is $L_{a} \cdot H_{i} / H$, the ionospheric term can be brought outside the integral. This suggests us to rewrite the ionosphere as: $\psi(\eta)=\psi_{m}+\psi_{d} \cdot \eta+\left(\psi(\eta)-\psi_{m}-\psi_{d}\right.$. $\eta)=\psi_{m}+\psi_{d} \cdot \eta+\psi_{r}(\eta)$, where $\psi_{m}$ is the average value of the ionosphere inside the aperture length projected at the ionosphere height $L_{i}$ and $\psi_{d}$ is the average derivative of the ionosphere:

$$
\begin{aligned}
& \psi_{m}=\frac{1}{L_{i}} \int \operatorname{rect}\left(\frac{\eta}{L_{i}}\right) \psi(\eta) d \eta \\
& \psi_{d}=\left.\frac{1}{L_{i}} \int \operatorname{rect}\left(\frac{\eta}{L_{i}}\right) \frac{d \psi\left(\eta^{\prime}\right)}{d \eta^{\prime}}\right|_{\eta^{\prime}=\eta} d \eta
\end{aligned}
$$

If $\psi_{r}(\eta)$ can be assumed negligible, Equation (2) becomes

$s_{f}(\eta)=a_{0} e^{j \alpha_{0}} e^{j \psi_{m}} \operatorname{sinc}\left(K L_{a}\left(\eta-\frac{K H_{i}}{f_{0}^{2} \cos \left(\theta_{i n c}\right)} \psi_{d}\right)\right)$,

while, if $\psi_{r}(\eta)$ becomes relevant the sinc function is blurred and the interferometric coherence drops. An accurate coregistration can corrects the shifts due to $\psi_{d}$, which also lower the coherence. If the ionosphere derivative is highly variable, however, it is possible that the shifts are not well estimated. Equation (4) shows how $\psi_{m}$ is the phase measured by an interferogram, the average of the ionosphere contained in the aperture. The target shift along azimuth gives an estimate of the average derivative of the ionosphere inside the aperture. In conclusion, a standard interferogram only shows the azimuth filtered low-pass portion of the ionosphere, while the high-pass portion lower the interferogram quality.

\section{PARTIAL FOCUSING}

In this section we derive the result of a partial focusing and demonstrate how it can expose the ionospheric phase screen and cancel the azimuth effects. Cosider the raw data acquisition as:

$$
\begin{aligned}
s_{r}(\eta)= & \int a(x) e^{j \alpha(x)} . \\
& \operatorname{rect}\left(\frac{\eta-x}{L_{a}}\right) e^{j \pi K(\eta-x)^{2}} e^{j \psi\left(\eta \frac{H_{i}}{H}+x \frac{H-H_{i}}{H}\right)} d x,
\end{aligned}
$$

where $a(x) e^{j \alpha(x)}$ is the ground reflectivity and phase, representing distributed scatterers. The focusing is performed using the following modified chirp:

$$
h_{s f}(\eta)=\operatorname{rect}\left(\frac{\eta}{L_{a}}\right) \exp \left(-j \pi K C \eta^{2}\right),
$$

where $C$ is a constant. The semi-focused data are thus

$$
\begin{aligned}
s_{s f}(\eta)= & \int s_{r}(u) h_{s f}(\eta-u) d u \\
= & e^{j \pi K C \eta^{2}} \int a(x) e^{j \pi K x^{2} .} \\
& \quad \int \operatorname{rect}\left(\frac{u}{L_{a}}\right) \operatorname{rect}\left(\frac{x-u}{L_{a}}\right) e^{j \phi(u) \eta} d u d x,
\end{aligned}
$$

where $\phi(u)$ is a phase term containing the chirp phase terms and the ionosphere:

$$
\begin{aligned}
\phi(u)=- & \pi K(C-1) \frac{u^{2}}{\eta}-2 \pi K u \frac{x}{\eta}+ \\
& 2 \pi K C u+\frac{1}{\eta} \psi\left(u \frac{H_{i}}{H}+x \frac{H-H_{i}}{H}\right) .
\end{aligned}
$$

Equation (7), which contains an integral of an oscillating phase term $\phi(u)$, can be solved using the stationary phase method. The stationary phase point $u_{0}$ can be found by searching the value of $u$ for which the phase derivative $\phi^{\prime}(u)$ is zero:

$$
\phi^{\prime}(u)=-2 \pi K(C-1) \frac{u}{\eta}-2 \pi K \frac{x}{\eta}+2 \pi K C+\psi^{\prime}(\ldots) \frac{H_{i}}{\eta H} .
$$

We assume that the derivative of the ionospheric phase term $\psi^{\prime}$ does not change the position of $u_{0}$ and that it can be neglected. By choosing $C$ equal to $H /\left(H-H_{i}\right)$ Equation (7) becomes:

$$
\begin{aligned}
s_{s f}(\eta) \approx & e^{j \pi K C \eta^{2}} \int a(x) e^{j \alpha(x)} e^{j \pi K x^{2}} . \\
& \operatorname{rect}\left(\frac{\eta-x}{L_{a} \frac{C-1}{C}}\right) e^{j \phi\left(u_{0}\right) \eta} e^{j \frac{\pi}{4}} d x \\
= & e^{j \psi(\eta)} e^{j \frac{\pi}{4}} \int a(x) e^{j \alpha(x)} \operatorname{rect}\left(\frac{\eta-x}{L_{i}}\right) . \\
& e^{j \pi K \frac{H}{H_{i}}(\eta-x)^{2}} d x .
\end{aligned}
$$

The ionospheric phase screen $e^{j \psi(\eta)}$ is now superimposed to the semi-focused data without being low-pass filtered and without to interfere with the impulse response function. The resolution of the semi-focused azimuth line of Equation (10) depends on the length of the aperture, projected at the ionosphere height. The gain factor of the focusing process is reduced because of the just partial focusing. Since this is counterbalanced by the increment of the resolution cell, the coherence does not change. The achievable accuracy of coregistration by cross-correlation also depends on the resolution cell, 
leading to a less precise coregistration accuracy measured in meters. Nevertheless, the coherence degradation due to miscoregistration is proportional to the resolution cell, therefore, since the accuracy measured in fractions of resolution cell is the same, we do not expect a loss of coherence. On the contrary, since the impulse response function is no more modified by the ionospheric phase, all azimuth effects are canceled, leading to an increase of the coherence. The more turbulent the ionosphere was, the bigger the increase is, therefore, the semi-focusing processing is only useful when the images are severely degraded by a turbulent ionosphere. Techniques like Delta-k or Phase-Group Delay Difference ([5]) can then be applied to separate the two components, topographic and ionospheric, of the interferometric phase and to obtain a highresolution estimation of the ionosphere.

Semi-focused images are in a sort of opposite plane with respect to focused images. The topographic phase, in fact, results low-pass filtered in semi-focused images because of the resolution decrease, while, to the ionospheric phase happens the opposite. Moreover, azimuth variations of the topographic phase generate shifts in semi-focused images like azimuth variations of the ionospheric phase do to focused images. A variation of half a cycle or more, inside the same resolution cell, reduces the coherence of semi-focused to zero. Therefore, the topographic and/or deformation phase has to be removed prior to defocusing. If an a priori knowledge of the ground phase cannot be obtained, an azimuth split-band processing of the semi-focused images could be implemented to avoid the coherence loss.

\section{NUMERICAL SIMULATIONS}

Simulations are used to test the effects of a turbulent ionosphere on InSAR and to test the presented algorithm. A ray tracing simulator with the parameters of the proposed mission Tandem-L is used to produce raw data azimuth lines using distributed targets as ground scene model. The turbulent ionospheric phase screen used in the simulations are produced by filtering white Gaussian noise with the power spectral density function ([6]):

$$
\Psi(f)=\frac{T}{\left(f_{0}^{2}+f^{2}\right)^{p / 2}},
$$

where $T$ is a scaling constant, $f_{0}$ is related to the outer scale length $l_{0}$ and $p$ is the spectral index. Typical parameters of the model for different test cases, considering strong and weak scintillation levels, have been generated using the Global Ionospheric Scintillation Model (GISM). In Figure 2 is shown an example of a generated phase screen in case of severe scintillation.

In the following are presented the results of the simulation done using the phase of Figure 2. In Figure 3 can be seen the coherence of focused data, represented with a black line, which is quite low due the azimuth shifts and blurring. The

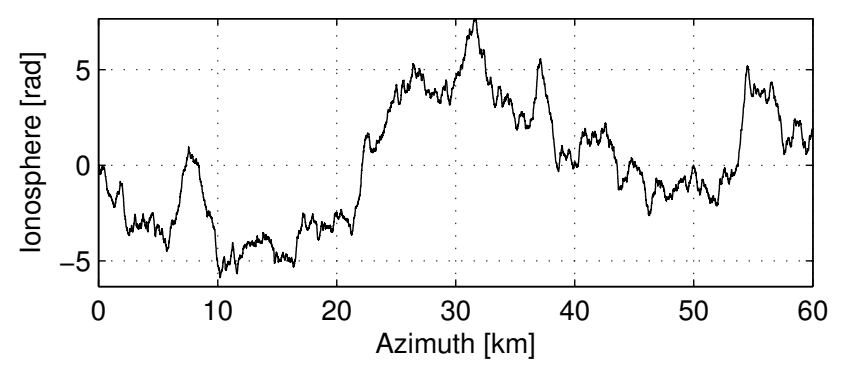

Fig. 2. Example of simulated ionospheric phase screen for strong scintillation condition.

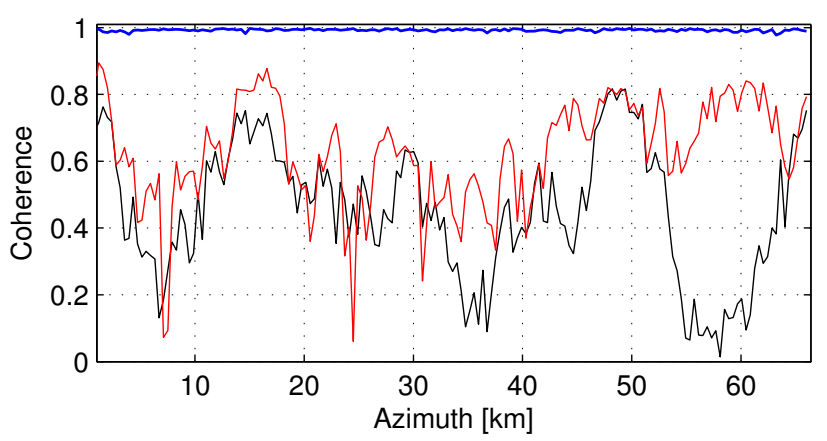

Fig. 3. Simulation example using the phase screen of Figure 2 , in black the coherence of the focused data before coregistration and, in red, after coregistration. In blue the coherence of the semi-focused data.

red line represents the coherence after the coregistration step. Since ionospheric azimuth effects are absent in semi-focused images, the coherence (blu line in Figure 3 ) is fully recovered.

Figure 4 shows the unwrapped interferogram from the focused and semi-focused data, along with the real and the lowpass filtered ionosphere. As expected, the interferogram is related to the low pass ionosphere, filtered by the aperture length. The interferogram from semi-focused, on the contrary, follows the true ionosphere.

\section{REAL DATA EXAMPLE}

A quad-pol PALSAR image pair is used to test the method on real data. The master image was acquired at 7:30 on April 1st 2007 and the slave on May 17th 2007. The coherence is quite low due to the vegetated terrain and due to a sudden change in the TEC level located in the top part of the image. The coherence, averaged along the range direction, is represented in black in Figure 5, while the coherence after the defocusing in blue. An improvement of the coherence can be seen in particular in areas where the ionosphere change was sudden and of high magnitude. This improvement is also visible comparing the two interferograms in Figure 6. The interferogram from semi-focused images, on the right, shows much more clearly 


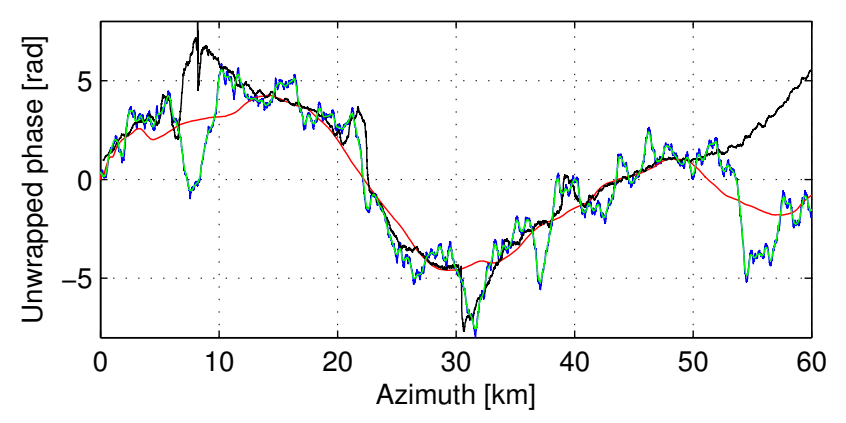

Fig. 4. Simulation example, interferogram of focused (black line) and semi-focused images (green line) along with ionosphere (blue line) and low-pass ionosphere (red line).

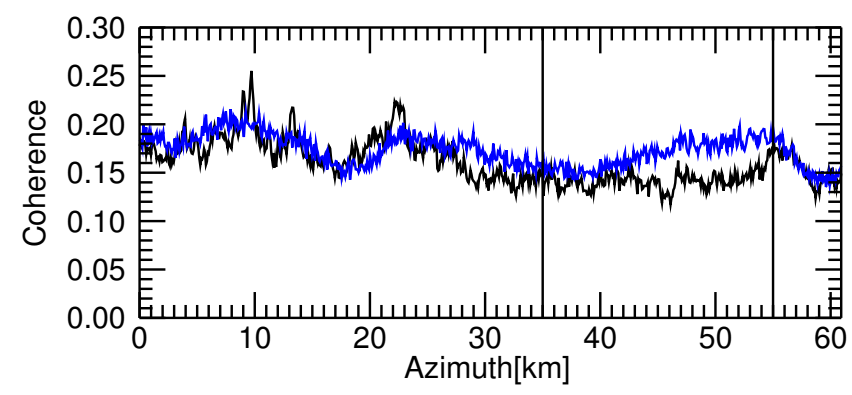

Fig. 5. Real data example. Black line: coherence of focused images pair. Blue line: coherence of semi-focused images. Inside the two vertical lines, the area where the ionosphere is steeper.

the fringes of the ionosphere steep slope.

\section{CONCLUSION}

The theoretical derivation of the semi-focusing technique, a simulation for a severe scintillation scenario and a real data example has been presented. It has been demonstrated that the semi-focusing can cancel azimuth effects and expose the true ionospheric phase screen. In case the images are disrupted by the electron density variations along the flight path, the semifocusing is able to recover the coherence permitting a better estimation and removal of the ionosphere.

\section{REFERENCES}

[1] A. Moreira, G. Krieger, M. Younis, I. Hajnsek, K. Papathanassiou, M. Eineder, and F. De Zan, "Tandem-l: A mission proposal for monitoring dynamic earth processes," in Geoscience and Remote Sensing Symposium (IGARSS), 2011 IEEE International, july 2011, pp. 1385 -1388 .

[2] M. Eineder, A. Friedrich, C. Minet, R. Bamler, F. Flerit,
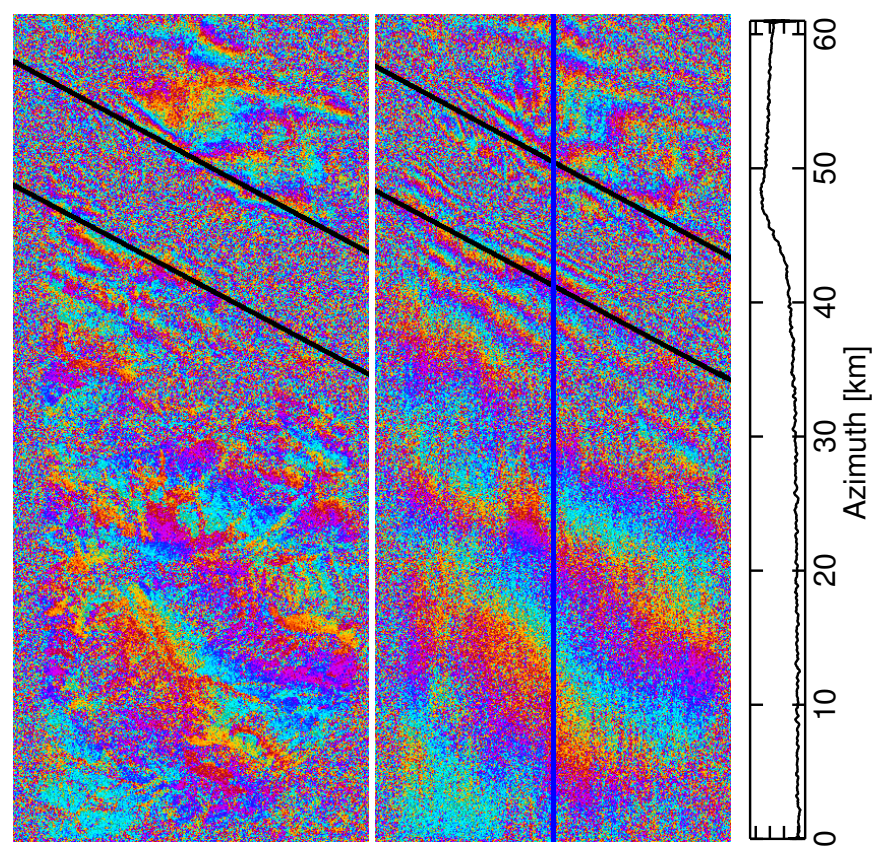

Fig. 6. Interferogram of focused images (left). Interferogram of semi-focused images (center). The black lines indicate the zone of the ionosphere TEC peak and steep slopes. On the right, the Faraday rotation calculated for the blue line zone, showing the ionosphere.

and I. Hajnsek, "Scientific requirements and feasibility on an 1-band mission dedicated to measure surface deformation," in Geoscience and Remote Sensing Symposium,2009 IEEE International,IGARSS 2009, 2009, vol. 2, pp. II-789-II-792.

[3] S. Tebaldini, A. Monti Guarnieri, and F. Rocca, "Recovering time and space varying phase screens through sar multi-squint differential interferometry," in Synthetic Aperture Radar, 2012. EUSAR. 9th European Conference on, 2012, pp. 16-19.

[4] J.S. Kim, K.P. Papathanassiou, S. Quegan, and N. Rogers, "Estimation and correction of scintillation effects on spaceborne p-band sar images," in Geoscience and Remote Sensing Symposium (IGARSS), 2012 IEEE International, 2012, pp. 5101-5104.

[5] R. Brcic, A. Parizzi, M. Eineder, R. Bamler, and F. Meyer, "Estimation and compensation of ionospheric delay for sar interferometry," in Geoscience and Remote Sensing Symposium (IGARSS), 2010 IEEE International, 2010, pp. 2908-2911.

[6] C. L. Rino, "A power law phase screen model for ionospheric scintillation: 1. weak scatter," Radio Science, vol. 14, no. 6, pp. 1135-1145, 1979. 